Sādhanā Vol. 40, Part 6, September 2015, pp. 1683-1699. (C) Indian Academy of Sciences

\title{
An improved predictive association rule based classifier using gain ratio and T-test for health care data diagnosis
}

\author{
M NANDHINI $^{1, *}$ and S N SIVANANDAM ${ }^{2}$ \\ ${ }^{1}$ Computer Science and Engineering, PSG College of Technology, \\ Coimbatore, 641004 India \\ ${ }^{2}$ Computer Science and Engineering, Karpagam College of Engineering, \\ Coimbatore, 641032 India \\ e-mail: nandumano@yahoo.co.in; sns12.kit@gmail.com
}

MS received 15 May 2014; revised 31 March 2015; accepted 24 April 2015

\begin{abstract}
Health care data diagnosis is a significant task that needs to be executed precisely, which requires much experience and domain-knowledge. Traditional symptoms-based disease diagnosis may perhaps lead to false presumptions. In recent times, Associative Classification (AC), the combination of association rule mining and classification has received attention in health care applications which desires maximum accuracy. Though several AC techniques exist, they lack in generating quality rules for building efficient associative classifier. This paper aims to enhance the accuracy of the existing CPAR (Classification based on Predictive Association Rule) algorithm by generating quality rules using Gain Ratio. Mostly, health care applications deal with high dimensional datasets. Existence of high dimensions causes unfair estimates in disease diagnosis. Dimensionality reduction is commonly applied as a preprocessing step before classification task to improve classifier accuracy. It eliminates redundant and insignificant dimensions by keeping good ones without information loss. In this work, dimensionality reductions by T-test and reduct sets (or simply reducts) are performed as preprocessing step before CPAR and CPAR using Gain Ratio (CPAR-GR) algorithms. An investigation was also performed to determine the impact of T-test and reducts on CPAR and CPAR-GR. This paper synthesizes the existing work carried out in AC, and also discusses the factors that influence the performance of CPAR and CPAR-GR. Experiments were conducted using six health care datasets from UCI machine learning repository. Based on the experiments, CPAR-GR with T-test yields better classification accuracy than CPAR.
\end{abstract}

Keywords. Associative classification (AC); Classification based on Predictive Association rule; gain ratio; dimensionality reduction; T-test; reduct Set.

${ }^{*}$ For correspondence 


\section{Introduction}

In recent years, health care data mining has gained its significance. Extracting interesting patterns using existing domain knowledge to support reasonable decision-making for the diagnosis and prognosis of disease becomes essential. Association rule mining is a descriptive data mining task commonly used for market-basket analysis. Description about the purchase behaviour of the customer and the relationship among market-basket items are uncovered by the association rules. An association rule is an implication of the form $\mathrm{X}->\mathrm{Y}$, where $\mathrm{X}$ and $\mathrm{Y}$ are item sets, which are disjoint in nature. Classification is a predictive data mining task used to determine a model using historical data to predict some response of interest. Associative Classification (AC) is a recent technique, which integrates the concept of classification and association rule mining. In literature, for health care data mining, Apriori and Frequent Pattern (FP) growth are the popular association rule mining algorithms used to extract Class Association Rules (CAR's). CAR's discover the inter-relationship between the disease symptoms that are useful for disease prediction. Though the associative classification is powerful classifier, it experiences poor classifier accuracy, since it generates large number of CAR's to construct the classifier. In this paper, CPAR-GR is proposed to improve the accuracy of the existing CPAR classifier for health care data diagnosis. At the same time, determining the quality of the rules is an important task. It can be identified by using appropriate error estimate measures such as Laplace accuracy and Likelihood ratio statistics. In this work, Laplace accuracy is employed for rule evaluation. Based on Laplace accuracy, the best k-rules are selected for classifier construction. Dimensionality reduction is a commonly used pre-processing step for any data mining task. Reducing the number of attributes by keeping the most significant ones always enhances the quality of the results. Consequently, retaining significant attributes in a dataset contributes high classifier accuracy. To achieve that, statistical T-test and reducts computation have been performed to identify the significant attributes from health care datasets. To conclude, the impact of the both dimensionality reduction techniques with CPAR and CPAR-GR has been analysed. From the experimental results, it is found that the combination of T-test and CPAR-GR yields better results in terms of classifier accuracy.

The rest of the paper is organized as follows. Section 2 discusses the literature survey. Section 3 describes the methodology followed in the proposed work. Experimental results are discussed in section 4 and section 5 includes conclusion and future scope.

\section{Background and related work}

Associative classification is one of the recent data mining techniques used in decision making for health care diagnosis (Antonie et al 2003; Jabbar et al 2012; Noh et al 2006). It combines the concepts of association and classification. Construction of an associative classifier involves the extraction of CAR from the training dataset and classification of the test dataset into predefined class labels using CAR.

\subsection{Associative classification}

Liu et al (1998), proposed Classification Based on Association (CBA), the first AC algorithm employed Apriori to generate the complete set of CAR's satisfying the user-specified minimum support and minimum confidence. The major drawback was that it performs a single rule based classification and it cannot handle large volume of rules. Li et al (2001), proposed Classification 
Based on Multiple Association Rules (CMAR) to overcome the drawback of CBA. It employed FP Growth algorithm for association rule mining phase. FP growth is one of the efficient rule mining algorithms and is the best alternative to Apriori. Unlike CBA, CMAR selects more than one rule from each class that satisfies a tuple. Finally, the class label of that tuple was determined using weighted Chi-square $\left(x^{2}\right)$ test. Though CMAR yields better classifier accuracy than CBA, it often generates a large number of rules in the rule mining phase thereby taking time and effort to choose interesting rules among them.

Several variants of predictive rule mining algorithms such as First Order Inductive Learner (FOIL) (Quinlan \& Cameron 1993), Predictive Rule Mining (PRM) (Yin \& Han 2003) and Classification based on Predictive Association Rule (CPAR) (Yin \& Han 2003) were proposed to overcome the problem of time complexity by generating small set of efficient predictive rules directly from the dataset rather than generating large set of candidate rules. FOIL is commonly used for binary class dataset; CAR's generated by FOIL learns to distinguish positive class tuples and negative class tuples. It employed a measure popularly known as FOIL's Information Gain to identify the best gain attribute to structure the rule antecedent. FOIL continuously searches for the best rule and keeps on removing all positive class tuples in the dataset that was satisfied by the best rule. The procedure was repeated until all the positive class tuples in the dataset are satisfied. The major drawback of FOIL was it did not achieve high accuracy as it generated a very small number of rules. It also suffered from time complexity since it always spends time in evaluating each and every attribute to find the best gain attribute. Yin \& Han (2003), proposed PRM which is an extension of FOIL to achieve higher accuracy and efficiency. Instead of removing all positive class tuples satisfied by the current best rule from the dataset, PRM reduces the weights of each positive class tuple in the dataset by a decay factor. PRM generates more rules as the positive class tuples and they were removed from the dataset only when the associated weights reached the gain threshold. PRM chooses only the best gain attribute to be included in the rule antecedent. Because of this, it misses other important rules that could be generated from closeto-the-best attributes.

CPAR proposed by Yin \& Han (2003), was an extension of PRM. Instead of selecting one best gain attribute, it selects all close-to-the-best attributes to be included in the rule antecedent using Gain_Similarity_Ratio (GSR). It uses an error estimate measure known as Laplace accuracy to evaluate each rule and it finally considers only the best k-rules in each class to predict the likelihood of the class of test tuple. The classifier build using CPAR achieved higher accuracy than FOIL and PRM.

\subsection{Dimensionality reduction}

In literature, several dimensionality reduction techniques are available for classifying high dimensional dataset. Reducing dimensions can increase the capability of extracting knowledge from high dimensional datasets. It also improves the classification accuracy by reducing computational cost of inducing classifiers. Broadly, dimensionality reduction techniques are divided into feature extraction and feature selection. Feature extraction methods are used to transform the dataset in a high dimensional space to a fewer dimensional space. Whereas, feature selection methods are used to select a subset of significant features (also called attributes or dimensions) that are useful for classifier construction. Based on the strategies, feature selection methods are categorized into two, namely, filter and wrapper.

For a classification task, with respect to the general characteristics like mutual information gain, correlation and distance to the class attribute, filter methods select and rank the significant features of a training dataset. Filter methods are more suitable for health care applications, since 
most of the health care applications deal with the storage and retrieval of high dimensional datasets. It is computationally efficient than wrapper methods. While wrapper methods require predetermined learning algorithm to evaluate and select the subset of significant features that gives best classifier performance. Classifier performance is wrapped on the ability of the learning algorithm which exploits dependencies among features in a subset.

In this paper, dimensionality reduction is performed as a pre-processing task before the associative classification algorithm (i.e., CPAR-GR). They are carried out in two methods namely, statistical T-test and by computing reducts. The former is a filter-based feature selection method and the latter is a wrapper-based feature selection method.

\subsection{Statistical T-test}

Lei \& Huan (2003), proposed a fast filter technique to identify the relevant attributes as well as redundancy among the relevant attributes from health care datasets. The drawback of this technique is that it selects very few attributes as significant that results in under fitting. Wang et al (2012), proposed a T-test based approach to measure the diversity of the distributions of each term in a text document between the specific category and the entire corpus. The paper concluded that T-test based feature selection produce better results than other feature selection methods such as Chi-Square $(\chi 2)$ statistic and Information Gain. Li et al (2006), used T-test to extract the gene features for building efficient classifier for cancer disease diagnosis. In this paper, input tuples were separated into two classes for computing t-score for each gene. Genes with t-scores greater than a set threshold were selected for classifier construction. Genes with t-scores less than a set threshold were considered as insignificant and thus they were removed from gene expression dataset. Wang et al (2007) introduced a feature importance ranking scheme using tscore to select very few significant genes. Using very few genes, an accurate classifier was built for cancer diagnosis. Poolsawad et al (2012) applied three different filter-based feature selection methods such as T-test, entropy and non-linear gain analysis to build a reliable prediction model for cardiology. In their work, it was concluded that T-test is the best feature selection method for binary class dataset as it follows normal distribution. It was suggested that the choice of feature selection method directly depends on the nature of the dataset.

\subsection{Rough set theory}

Rough set theory introduced by Pawlak $(1982,1991)$ is a formal methodology employed for feature selection using reduct sets. A reduct set (also known as reduct) is a minimal set of attributes that is sufficient to represent the class structure. Determining all possible reducts from a dataset is a NP-complete problem (Susmaga 2004). In literature there were many approximation algorithms, including greedy algorithms (Johnson 1974) and genetic algorithms (Vinterbo \& OhnoMachado 1999) used to find reducts. In Pila \& Monard (2001), the set of experiments were carried out for feature subset selection using rough set reducts along with other filter methods. The exhaustive reducer algorithm proposed in (Komorowski et al 2002; Øhrn 1999), generates all possible reducts by brute force, using exhaustive search. This algorithm takes exponential time in terms of number of objects and it was not suitable for very large decision systems. Vinterbo \& Ohno-Machado (1999), proposed a Genetic Algorithm (GA) based feature selection method for a classification task. This technique generates the maximum possible reducts thereby solving the minimal cover set problem. Qiang \& Alexios (2001), discusses the benefits of the Rough Set Attribute Reduction (RSAR) technique for both supervised and unsupervised learning. Li \& Nick (2005) introduced a new method to discover and rank the association rules using rough set 
theory. In their work, the decision rules were generated from each reduct of a dataset instead of generating association rules from original dataset. This paper concluded that rules generated from reducts i.e., reduct rules were found to be more interesting than ordinary rules. Kohavi \& Frasca (1994) demonstrated that a classifier using simple decision table from rough set theory offered good results on artificial and real-world datasets. Jiao \& Miao (2009) proposed an efficient gene selection algorithm using Tolerance Rough Set Theory. In this work, the performance of the traditional rough set theory was compared with the tolerance rough set theory. It was concluded that the tolerance rough set theory is well-suited for gene expression dataset which is of continuous in nature where as traditional rough set theory does not handle continuous values. Rough set theory has made a significant contribution in medical data diagnosis (Hassanien et al 2009; Pila \& Monard 2001).

\section{Methodology}

The proposed methodology incorporates three major phases: dimensionality reduction, associative classifier construction and test tuple classification. In the first phase, dimensionality reduction by retaining significant attributes and eliminating insignificant attributes are performed using T-test and reducts computation. The second phase involves the construction of Associative Classifier (AC). Using the CPAR-GR, class rules i.e., CAR's are generated. Once the class rules are generated, class rules are ordered based on the Laplace accuracy value. Laplace accuracy is one of the class rules evaluation metric used in FOIL (Quinlan \& Cameron 1993), PRM (Yin \& Han 2003) and CPAR (Yin \& Han 2003) which determines the quality of the class rules by considering its rule coverage. In the final phase, the best k-rules from each class that satisfies the given test tuple are selected according to the Laplace accuracy. Average Laplace accuracy of the best k-rules from each class is determined. A class with the highest Laplace average accuracy is chosen as the class label for the test tuple. The detailed work flow involved in this methodology is outlined in figure 1.

\subsection{Preprocessing the dataset}

Health care dataset consists of continuous valued attributes which cannot be directly taken for processing. With Weka 3.7 the continuous valued attributes are discretized using discretize filter. Discretization is performed by simple binning with findNumBins set as False, and number

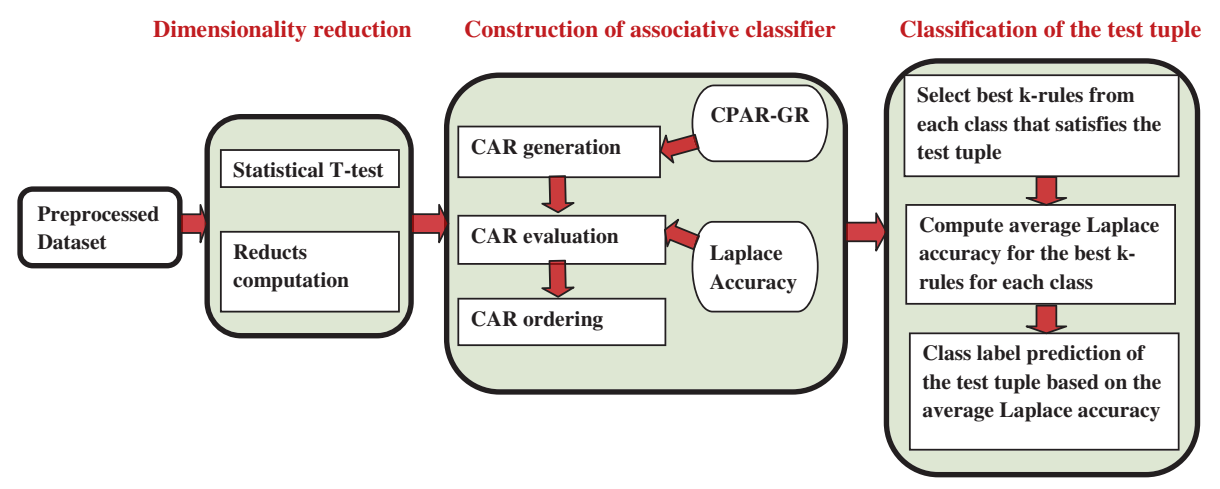

Figure 1. Workflow of the proposed methodology. 
of bins as 10. Even the missing values for nominal and numeric attributes in a dataset are also replaced with the modes and means from the training data. Specialty of health care data lies in the fact that the attribute values can only be within certain ranges. All the six health care datasets are separately preprocessed using Weka 3.7 according to the requirements of the designed system.

\subsection{Dimensionality reduction by T-test (DRT)}

The quality of class rules plays a vital role in the construction of an efficient classifier. It can be improved by performing dimensionality reduction using T-test. The statistical T-test is a filter method commonly used to select the important features having higher significance towards classification. Statistical T-test, an unpaired T (also known as student's T-test) is performed using Mattest function, in MATLAB 7.0.1. It computes p-value and t-score for each attribute. Attributes with the p-value less than or equal to 0.05 are considered as significant. The attributes which are less significant (i.e., greater than 0.05) are removed from the dataset.

\subsection{Dimensionality reduction by reducts (DRR)}

In rough set theory (Qiang \& Alexios 2001), most of the attribute selection algorithms are reduct-oriented. A reduct is a minimal attribute (feature) subset that preserves an indiscernibility relation. Such a relation may be formulated either for the full system or related to a class of object. Generally, the approaches of reducts computation are classified into two; discernibility function-based and attribute dependency-based. ROSETTA (ROugh SET Toolkit for Analysis of data) is a GUI environment which supports knowledge discovery and data mining within the structure of rough set theory. This environment is planned to support the entire knowledge discovery process starting from data pre-processing, to validation and analysis of the results. In this work, using Genetic Reducer algorithm in ROSETTA 1.4.41, discernibility function-based reducts are computed from each of the six health care datasets.

\subsection{Associative classifier construction}

Six datasets after dimensionality reduction are taken into classifier construction. CPAR-GR is an associative classifier which employs significant positive and negative class rules to build a model. CPAR-GR takes the fundamental idea of FOIL, PRM and CPAR to generate class rules. Initially, the dataset is partitioned into positive and negative set. At each iteration, each attribute is evaluated using Gain Ratio and the best gain attribute among the attributes of the dataset is added to the rule antecedent to form class rules. Gain value of an attribute with respect to positive and negative set is calculated using Eq. (1). After including the attribute, the tuples matching it will be removed from the dataset. This procedure is repeated until all the tuples of the dataset are covered. At the outset CPAR-GR generates all possible class rules from positive set; soon after the same procedure is repeated for negative set to generate negative class rules. During the rule generation, CPAR-GR selects all the Close-to-the-Best attributes in the rule antecedent instead of one best gain attribute so that it will generate more than one rule at the same time. Existing associative classification algorithms like PRM selects only one best gain attribute in the rule antecedent and ignore all the others. However, in a dataset there may be few attributes with gain values similar or close to the highest gain value and hence it misses some significant rules.

Table 1 shows the algorithm used for the CAR generation. Initially, the given dataset is partitioned into positive and negative sets. Total_Weight_Threshold (TWT) for positive set is 
calculated using Eq. (7). If the sum of weight of all the positive tuples is greater than the TWT, the iteration proceeds to the calculation of gain value for each attribute, else the current iteration stops and the same procedure is repeated for the negative set.

Existing CPAR algorithm employs FOIL's Information Gain measure as shown in Eq. (6) to seek the best gain attribute whereas CPAR-GR employs the Gain Ratio measure.

Local_Gain_Threshold (LGT) is a parameter used to determine all possible Close-to-theBest attributes. LGT is calculated using Eq. (8). The attributes with gain values which are

Table 1. Algorithm for CAR generation.

\section{Algorithm : CAR Generation}

// P'- to store the copy of P, N'- to store the copy of N.

// Gain_Ratio[n] - array to store gain value of 'n' attributes using Gain ratio

// Best_Gain - parameter used to hold the highest gain value.

// Close-to-the-Best[ ] - array to store the attributes with gain values greater than LGT.

\section{Input:}

- $\quad \mathrm{D}[\mathrm{n}]$-is the binary class dataset contains ' $\mathrm{n}$ ' attributes.

- $\quad$ P- set of positive tuples (i.e. records belong to positive class in D).

- N- set of negative tuples (i.e. records belong to negative class in D)

- $\quad \sum \mathrm{W}(\mathrm{P})$-sum of weights of all positive tuples, initially, weight of each tuple is 1.0.

- MGV- user specified minimum gain value.

- TWT- minimum weight threshold for P.

- LGT-Gain threshold for each attribute,

- CT- tuple coverage threshold.

- Decay_Factor - used to reduce the weight of the tuple.

Output: Class Association Rules (CAR's)

\section{Method:}

//Initialization of parameters

(1) Best_Gain $=0$;

(2) $\mathrm{TWT}=\Sigma \mathrm{W}(\mathrm{P}) * \mathrm{CT}$;

(3) While $\left(\sum \mathrm{W}(\mathrm{P})>\mathrm{TWT}\right)$

(4) $\quad \mathrm{P}^{\prime} \leftarrow \mathrm{P}, \mathrm{N}^{\prime} \leftarrow \mathrm{N}$; $\quad$ //create a copy of $\mathrm{P}$ and $\mathrm{N}$ in $\mathrm{P}^{\prime}$ and $\mathrm{N}^{\prime}$

(5) for $\mathrm{i}=1$ to $\mathrm{n}$ do //For each attribute of $\mathrm{D}$.

(6) Calculate Gain_Ratio[i] //Calculate gain value of each attribute with respect to P'and N' using Eq. (1).

(7) if (Gain_Ratio[i] >= Best_Gain ) then

(8) Best_Gain = Gain_Ratio[i];

(9) end if

(10) end for

(11) LGT =Best_Gain *GSR;

(12) for $\mathrm{i}=1$ to $\mathrm{n}$ do

end for

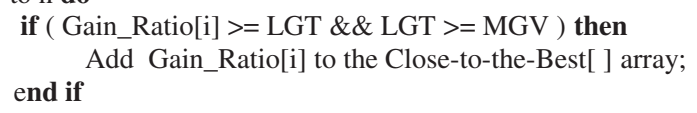


close/similar to LGT are considered as Close-to-the-Best attributes. Gradually, class rules are formed by adding the Close-to-the-Best attributes in the rule antecedent. Tuples that do not contain the current Close-to-the-Best attribute that has been included are removed from the positive and negative set. If negative set is empty, rule antecedent $\rightarrow$ positive class is added as a class rule in rule list. Simultaneously, weight of the positive tuple covered by the rule is decreased by Decay_Factor. The iteration continues until the total weight of all the positive tuples in the dataset satisfies the TWT. After the generation of all possible class rules from positive set, the same procedure is repeated to generate negative class rules by keeping the negative set as positive.

Coverage_Threshold (CT) is another important parameter used to determine the existence of the tuple in the training dataset. A tuple can be removed from the training set only if it satisfies a certain coverage threshold. Use of CT improves classifier accuracy. Including all Close-tothe-Best attributes in the rule antecedent makes the rule generation process faster. Finally, all possible predictive rules (i.e., CAR's) are generated from each class and they are further taken for classification.

\section{Gain ratio}

$$
\begin{array}{r}
\text { Gain Ratio }(A)=\operatorname{Gain}(A) / \text { SplitInfo }(A) \\
\text { SplitInfo }(D)=-\sum_{j=1}^{v} \frac{\left|D_{j}\right|}{|D|} * \log _{2}\left(\left|\frac{D_{j}}{D}\right|\right)
\end{array}
$$

where

$|D|$ represents the total number of tuples in the training dataset.

$\left|D_{j}\right|$ represents the total number of positive or negative tuples in the training dataset.

$' v$ ' represents the total number of classes in the dataset.

' $A$ ' represents the attribute to be included in the rule antecedent,

where $\operatorname{Gain}(A)$ is mutual information gain of the attribute ' $A$ ' which is calculated using Eq. (3).

$$
\begin{gathered}
\operatorname{Gain}(A)=I(p, n)-E(A) \\
E(A)=-\sum_{i=1}^{m} \frac{p_{i}+n_{i}}{p+n} I\left(p_{i}, n_{i}\right) \\
I(p, n)=-\frac{p}{p+n} \log _{2} \frac{p}{p+n}-\frac{n}{p+n} \log _{2} \frac{n}{p+n},
\end{gathered}
$$

where,

$E(A)$ represents the entropy of the attribute ' $A$ ' in the training dataset .

$I(p, n)$ represents the expected information needed to satisfy a given training dataset.

' $p$ ' represents the total number of positive tuples in the training dataset.

' $n$ ' represents the total number of negative tuples in the training dataset.

' $p_{i}^{*}$ ' represents the number of positive tuples covered by the $\mathrm{i}^{\text {th }}$ value of the attribute ' $A$ ', which is to be included in the rule-antecedent.

' $n_{i}^{*}$ ' represents the number of negative tuples covered by the $\mathrm{i}^{\text {th }}$ value of the attribute ' $A$ ', which is to be included in the rule-antecedent.

' $m$ ' represents the number of values for an attribute ' $A$ '.

\section{FOIL's information gain}

$$
\text { FOIL's Gain }(A)=\left|P^{*}\right| *\left(\log \frac{\left|P^{*}\right|}{\left|P^{*}\right|+\left|N^{*}\right|}-\log \frac{|P|}{|P|+|N|}\right),
$$


where

$P \mid$ represents the number of positive tuples covered by the current rule antecedent and consequent in the training dataset.

$\left|P^{*}\right|$ represents the number of positive tuples covered by the new rule antecedent (i.e., after including the best gain attribute in the rule antecedent) and consequent in the training dataset.

$|N|$ represents the number of negative tuples covered by the current rule antecedent and consequent in the training dataset.

$\left|N^{*}\right|$ represents the number of negative tuples covered by the new rule antecedent (i.e., after including the best gain attribute in the rule antecedent) and consequent in the training dataset. ' $A$ ' represents the attribute in the training dataset .

$$
T W T=\sum_{i=1}^{n} W\left(P_{i}\right) * C T,
$$

where

' $n$ ' represents the total number of positive tuples in the training dataset.

$W(\mathrm{Pi})$ represents the weight of the positive tuples in the training dataset.

$C T$ represents Coverage_Threshold.

$$
L G T=\text { Best_Gain } * G S R,
$$

where

Best_Gain represents the highest gain value.

GSR represents Gain_Similarity_Ratio.

\subsection{Test tuple classification}

Table 2 shows the algorithm used for classifying the test tuples. Algorithm describes the rule evaluation process carried out in this work. After the generation of high quality rules using Gain Ratio, the coverage of each rule over the dataset is evaluated using Laplace accuracy as per Eq. (9). Based on the Laplace accuracy, the class rules are arranged in descending order.

$$
\text { Laplace Accuracy }=\frac{(n c+1)}{(n t o t+k)}
$$

where

' $k$ ' represents the number of classes.

' $n c$ ' represents the total number of tuples in the training dataset satisfying both antecedent and consequent parts of the CAR.

'ntot' represents the total number of tuples in the training dataset satisfying antecedent part of the CAR.

\subsection{Best $k$-rule prediction}

The class rules that satisfy the test tuples are selected. The set of rules which matches every test tuple is identified and stored separately based on the class label. Among the entire set of rules, only the best $\mathrm{k}$ rules having the highest Laplace accuracy value are selected for predicting the class label of the test tuple. In this work, $\mathrm{k}$ is set as 5 i.e., top 5 rules having the highest Laplace accuracy value are selected from each class. 
Table 2. Algorithm for test tuple classification.

\begin{abstract}
Algorithm: Test_tuple classification
// R[m] - array used to store generated ' $\mathrm{m}$ ' class rules.

$/ / \mathrm{P}[\mathrm{n}]$ - array to store predicted class label of ' $\mathrm{n}$ ' test tuples.

//correct - parameter to store the count of correctly classified test tuples.

//wrong - parameter to store the count of incorrectly classified test tuples.

$/ / \mathrm{k}$ - Number of rules to be selected for test tuple classification.

//temp_P[ ] - temporary array to store positive class rules.

//temp_N[ ] - temporary array to store negative class rules.
\end{abstract}

\title{
Input:
}

- $\quad T[n]-$ is the binary class test dataset contains ' $n$ ' tuples.

- $\mathrm{R}[\mathrm{m}]$ - array contains generated ' $\mathrm{m}$ ' class rules.

- $\mathrm{A}[\mathrm{n}]-$ array contains actual class label of ' $\mathrm{n}$ ' test tuples.

Output: Accuracy of the classifier

Method:

//Initialization of parameters

(1) $\mathrm{k}=5$, correct $=0$, wrong $=0$;

(2) for $\mathrm{i}=1$ to $\mathrm{m}$ do $\quad$ //for each rule in $\mathrm{R}$

(3) Calculate Laplace accuracy for each R[i]

(4) end for

(5) Sort the $\mathrm{R}[\mathrm{m}]$ in descending order based on its Laplace accuracy value ;

(6) for $\mathrm{i}=1$ to $\mathrm{n}$ do //for each test tuple in $\mathrm{T}$

(7) for $\mathrm{j}=1$ to $\mathrm{m}$ do //for each rule in $\mathrm{R}$

(8) if ( antecedent.R[j] $\subset \mathrm{T}[\mathrm{i}] \& \&$ consequent. $R[\mathrm{j}]==$ 'Positive' $)$ then

(9) Store the $\mathrm{R}[\mathrm{j}]$ in temp_P[ ];

(10) end if

$$
\text { if }(\text { antecedent. } R[j] \subset T[i] \& \& \text { consequent. } R[j]==\text { 'Negative' }) \text { then }
$$

Store the R[j] in temp_N[ ]; end if

end for

select the best-k rules from temp_P[ ] and temp_N[ ];

calculate the average Laplace accuracy for each best-k class rules.

Class which has maximum average value is assigned as $\mathrm{P}[\mathrm{i}]$;

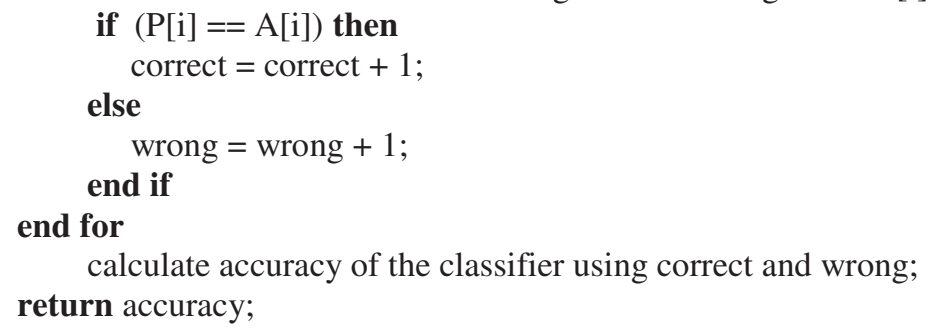

calculate accuracy of the classifier using correct and wrong; return accuracy;

\subsection{Evaluation of classifier accuracy}

A class which has maximum average accuracy is taken as the class label for the test tuple. This predicted class label is then compared with the actual class label of the test tuple. 
Finally, based on the number of correct classifications, the accuracy of the CPAR-GR is evaluated using Eq. (10). In this work, the accuracy of the classifier is evaluated using 50:50 percentage split test option (i.e., the entire dataset is equally split into training and testing dataset).

$$
\text { Accuracy }=(\text { No. of correctly classified tuples / Total no. of tuples })
$$

\section{Experiments and results}

The experiments were conducted using six health care datasets such as heart, hepatitis, wisconsin breast cancer (original), cleve, pima and sick from the UCI machine learning repository. As a sample breast cancer dataset is discussed in detail. Breast cancer dataset is commonly used to predict the cancer disease for a given set of patient's signs and symptoms. It consists of 11 attributes, where the $11^{\text {th }}$ attribute is a class attribute used to predict the kind/severity of the cancer (benign/malignant). Table 3 shows the attributes and its domain values of the breast cancer dataset. Table 4 lists the typical class rules generated on breast cancer dataset. Initially the six datasets are preprocessed by categorizing the attributes values based on the domain. As per the requirements of the algorithm, data preprocessing is carried out to transform the entire non class attribute values into nominal attributes (section 3.1). Preprocessed datasets are then taken into DRT using Mattest in MATLAB 7.0.1. Significant attributes obtained from the six health care datasets using Mattest function are shown in table 5. While performing T-test for the breast cancer dataset, most of the attributes are found to be significant. For Cleve dataset, the number of non-class attributes is 13 , after performing T-test, it is found that only 8 attributes have p-vlaue greater than 0.05 (at 95\% significance level). These 8 attributes provide sufficient information useful for an efficient classification. Similarly, DRT is applied for the remaining datasets.

Simultaneously, using ROSETTA, GUI version 1.4.41, the preprocessed dataset is taken for reducts computation (i.e., DRR). Table 6 , shows the various reducts obtained from the six health care datasets. From table 6, it is found that datasets such as hepatitis, pima and sick have only one reduct set which is directly taken as its significant features, whereas multiple reducts are generated for the datasets like breast cancer, cleve and heart. When multiple reducts are generated, choosing a valuable reduct becomes difficult. In order to compute single reduct from these

Table 3. Attributes of the breast cancer dataset.

\begin{tabular}{ll}
\hline Attribute & Domain \\
\hline Sample code number & Numeric, values ranges from 1-10 \\
Clump thickness & Numeric, values ranges from 1 - 10 \\
Uniformity of cell size & Numeric, values ranges from 1 - 10 \\
Uniformity of cell shape & Numeric, values ranges from 1 - 10 \\
Marginal adhesion & Numeric, values ranges from 1 - 10 \\
Single epithelial cell size & Numeric, values ranges from 1-10 \\
Bare nuclei & Numeric, values ranges from 1-10 \\
Bland chromatin & Numeric, values ranges from 1 - 10 \\
Normal nucleoli & Numeric, values ranges from 1 - 10 \\
Mitoses & Numeric, values ranges from 1 - 10 \\
Class & (Benign, Malignant) \\
\hline
\end{tabular}


Table 4. Typical class rules generated on breast cancer dataset.

R1: Clump_Thickness $=5$, Uniformity_Cell_Size $=1 \rightarrow$ Class $=$ Benign

R2: Marginal_Adhesion $=4$, Bare_Nuclei $=2$, Mitosis $=1 \rightarrow$ Class $=$ Benign

R3: Single_Epithelial_Cell_Size $=10$, Mitosis $=3 \rightarrow$ Class $=$ Malignant

R4: Uniformity_Cell_Shape $=9$, Bare_Nuclei $=6 \rightarrow$ class $=$ Malignant

datasets, reducts are computed after DRT i.e., T-test is followed by reducts computation. Initially T-test is applied over the datasets, based on the t-scores, significant attributes are retained by eliminating irrelevant attributes from the datasets.

Using genetic reducer algorithm in ROSETTA, the reducts are generated from the reduced dataset (i.e., dataset after T-test). Computation of reducts after DRT quickly generates the single reduct from each dataset. Table 7 shows the single reduct set obtained from three health care datasets such as breast cancer, cleve and heart. Finally, the significant features obtained by DRT, DRR and Single Reduct (SR) are taken into CPAR-GR separately for classifier construction. Generation and evaluation of class rules are performed in CPAR-GR as explained in sections 3.4-3.7. For classifier construction, the default parameters values used in CPAR-GR are taken from existing CPAR algorithm. CPAR-GR uses the parameters such as CT, MGV, GSR and Decay_Factor with the initial values of $0.05,0.7,0.99$ and $2 / 3$, respectively.

Table 8 shows the results of CPAR and CPAR-GR over the datasets with and without dimensionality reduction using 50:50 test option. From table 8 it is found that, for first three datasets such as breast cancer, cleve and hepatitis datasets, CPAR without dimensionality reduction yields better accuracy than CPAR-GR whereas CPAR-GR is better for the remaining three datasets. Results prove that the use of dimensionality reduction techniques have shown improvement in the accuracy of the CPAR algorithm for only three datasets such as cleve, heart and pima datasets whereas the CPAR-GR with dimensionality reduction shows improvement in accuracy for almost all datasets except hepatitis.

In particular, the combination of T-test and CPAR-GR has achieved a classification accuracy of $86.8 \%, 79.22 \%, 82.55 \%$ and $93.89 \%$ approximately $4 \%, 1 \%, 4 \%$ and $11 \%$ higher than the accuracy achieved using CPAR with T-test for cleve, hepatitis, pima and sick datasets, respectively. There is a negligible change in classifier accuracy for breast cancer and heart datasets, because T-test has identified almost all attributes as significant (table 5) and there is no effective dimensionality reduction taken place for these datasets. Also, the computation of reducts does not support the performance of the CPAR and CPAR-GR for all datasets except cleve and heart. On analysing facts, it is found that cleve and heart datasets are ended with the optimal average

Table 5. Significant attributes obtained from six health care datasets using T-test.

\begin{tabular}{llll}
\hline Dataset & $\begin{array}{l}\text { No. of non-class } \\
\text { attributes } \\
\text { before T-test }\end{array}$ & $\begin{array}{l}\text { Significant attributes (Integer } \\
\text { values represents the } \\
\text { attribute of a dataset) }\end{array}$ & $\begin{array}{l}\text { No. of non-class } \\
\text { attributes } \\
\text { after T-test }\end{array}$ \\
\hline Breast cancer & 10 & $2,3,4,5,6,7,8,9,10$ & 9 \\
Cleve & 13 & $1,2,7,9,10,11,12,13$ & 8 \\
Hepatitis & 19 & $2,4,5,6,10,11,12,13,14,18,19$ & 11 \\
Heart & 13 & $1,2,3,5,7,9,10,11,12,13$ & 10 \\
Pima & 8 & $1,2,3,5,6,7,8$ & 7 \\
Sick & 28 & $3,5,6,7,8,10,11,14,17,18,19,20,21,22,23,24,25,26$ & 18 \\
\hline
\end{tabular}


Table 6. Reducts computed using genetic reducer algorithm in ROSETTA for six health care datasets.

\begin{tabular}{|c|c|c|}
\hline Dataset & $\begin{array}{l}\text { No. of reduct } \\
\text { sets obtained }\end{array}$ & $\begin{array}{l}\text { Reduct sets obtained (integer value represents } \\
\text { the attribute of a dataset) }\end{array}$ \\
\hline Breast cancer & 20 & $\begin{array}{l}\text { R1: } 2,5,7,8 \\
\text { R2: } 2,3,7,9 \\
\text { R3: } 4,6,7,8 \\
\text { R4: } 2,4,7,9 \\
\text { R5: } 2,4,6,7 \\
\text { R6: } 2,6,7,9 \\
\text { R7: } 2,3,7,8 \\
\text { R8: } 2,3,4,5,7 \\
\text { R9: } 3,4,5,7,9 \\
\text { R10: } 3,6,7,8,10 \\
\text { R11: } 2,4,5,7,8 \\
\text { R12: } 4,5,7,8,10 \\
\text { R13: } 2,5,6,7,8 \\
\text { R14: } 3,6,7,8,9 \\
\text { R15: } 4,5,6,7,9 \\
\text { R16: } 2,4,5,7,10 \\
\text { R17: } 2,3,5,7,10 \\
\text { R18: } 4,5,7,9,10 \\
\text { R19: } 2,3,6,7,10 \\
\text { R20: } 6,7,8,9,10\end{array}$ \\
\hline Cleve & 7 & $\begin{array}{l}\text { R1: } 1,3,4,5,7,8,9,11,12,13 \\
\text { R2: } 1,3,4,7,8,9,10,11,12,13 \\
\text { R3: } 1,2,3,4,6,7,8,9,12,13 \\
\text { R4: } 1,3,4,6,7,8,9,11,12,13 \\
\text { R5: } 1,2,3,4,5,6,7,8,9,10,13 \\
\text { R6: } 1,2,3,4,5,7,8,9,10,12,13 \\
\text { R7: } 1,3,4,5,6,7,8,9,10,11,13\end{array}$ \\
\hline Hepatitis & 1 & $\mathrm{R} 1: 1,6,8,9,11,14,17,19$ \\
\hline Heart & 7 & $\begin{array}{l}\text { R1: } 1,3,4,7,8,10,11,12,13 \\
\text { R2: } 1,3,4,5,7,8,11,12,13 \\
\text { R3: } 1,2,3,4,6,7,8,12,13 \\
\text { R4: } 1,3,4,6,7,8,11,12,13 \\
\text { R5: } 1,2,3,4,5,7,8,10,12,13 \\
\text { R6: } 1,3,4,5,6,7,8,9,10,11,13 \\
\text { R7: } 1,2,3,4,5,6,7,8,9,10,13\end{array}$ \\
\hline Pima & 1 & $\mathrm{R} 1: 1,2,3,4,5,6,7,8$ \\
\hline Sick & 1 & R1: $1,2,3,4,5,6,8,9,10,11,12,13,14,16,17,18,20,22,23,24,26,28$ \\
\hline
\end{tabular}

Table 7. Single reduct obtained from three health care datasets such as breast cancer, cleve and heart.

\begin{tabular}{lll}
\hline Dataset & $\begin{array}{l}\text { Reduct set obtained (integer value represents } \\
\text { the attribute of the dataset) }\end{array}$ & $\begin{array}{l}\text { No. of attributes in the } \\
\text { single reduct set }\end{array}$ \\
\hline Breast cancer & R1: $5,6,7,8,9$ & 5 \\
Cleve & R1: $1,2,7,9,10,11,12,13$ & 8 \\
Heart & R1:1,2,3,5,7,9,10,11,12,13 & 10 \\
\hline
\end{tabular}


Table 8. Comparison of CPAR and CPAR-GR algorithms in terms of classifier accuracy (\%).

\begin{tabular}{|c|c|c|c|c|c|c|c|c|}
\hline \multirow[b]{2}{*}{ Dataset } & \multicolumn{4}{|c|}{ CPAR } & \multicolumn{4}{|c|}{ CPAR-GR } \\
\hline & $\overline{\text { WDR }}$ & $\overline{D R R}$ & DRT & SR & $\overline{\mathrm{WDR}}$ & DRR & DRT & $\overline{S R}$ \\
\hline Breast Cancer & 95.7 & $33.91^{*}$ & 95.7 & 33.48 & 95.13 & $33.33^{*}$ & 95.13 & 33.76 \\
\hline Cleve & 82.78 & $88.45^{*}$ & 82.84 & 82.84 & 79.47 & $84.82 *$ & 86.8 & 86.8 \\
\hline Hepatitis & 84.42 & 74.03 & 77.92 & 77.92 & 81.82 & 79.22 & 79.22 & 79.22 \\
\hline Heart & 75.56 & $88.52 *$ & 87.04 & 87.04 & 78.52 & $83.7 *$ & 87.04 & 87.04 \\
\hline Pima & 70.31 & 70.31 & 78.52 & 78.52 & 72.14 & 72.14 & 82.55 & 82.55 \\
\hline Sick & 84.86 & 82.64 & 83.25 & 72.5 & 91.5 & 91.36 & 93.89 & 93.89 \\
\hline Average accuracy(\%) & 82.27 & 72.97 & 84.21 & 72.05 & 83.09 & 74.09 & & 77.21 \\
\hline
\end{tabular}

WDR (Without Dimensionality Reduction) i.e., Dataset with complete set of attributes

DRR (Dimensionality Reduction by Reducts) i.e., Reduced dataset with significant attributes obtained by reducts

DRT (Dimensionality Reduction by T-test) i.e., Reduced dataset with significant attributes obtained by T-test

SR (Single Reduct) i.e., Reduced dataset with significant attributes of the single reduct

*represents the average classifier accuracy achieved using all reducts

reduct size, whereas the average reduct size of breast cancer dataset is 4.6 almost loosing all its significant attributes.

In this paper, performance of CPAR-GR over single reduct obtained from each dataset is also analysed. Though the generation and computation of single reduct (i.e., by computing reducts after T-test) is made simple, it has not achieved better accuracy than T-test with CPAR-GR. As part of this work, an investigation on the factors that influence the performance of CPAR-GR has been done. Most of the attributes of these six datasets considered in this paper are naturally significant and cannot be ignored, hence the scope for dimensionality reductions over these

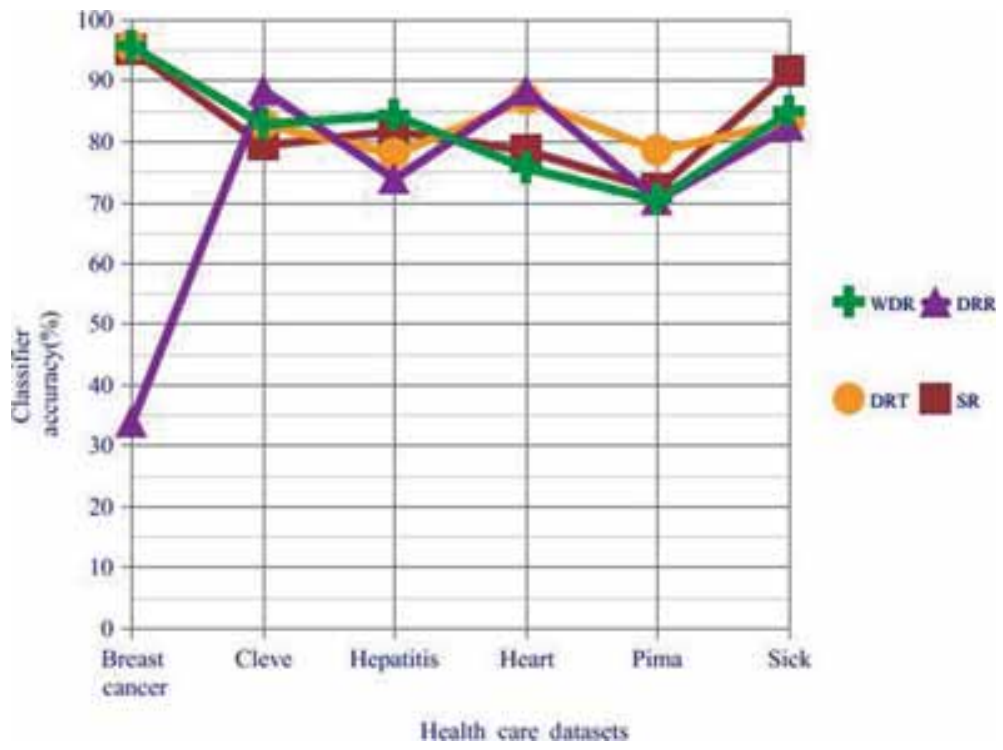

Figure 2. Performance of the CPAR algorithm over six health care datasets with and without dimensionality reduction techniques. 


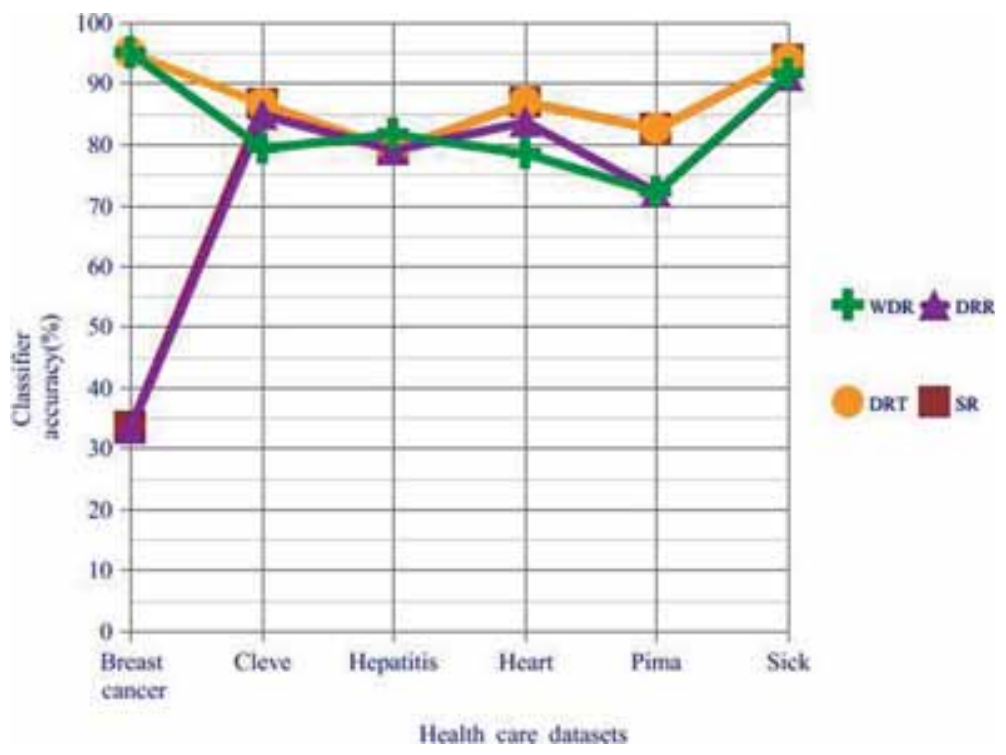

Figure 3. Performance of the CPAR-GR algorithm over six health care datasets with and without dimensionality reduction techniques.

datasets are very minimal. If the CPAR-GR is applied on the datasets which require dimensionality reduction to a greater extent, it would definitely yield higher accuracy than that is obtained in this paper. The accuracy of the CPAR and CPAR-GR over the six health care datasets with and without dimensionality reductions are represented as line graphs in figures 2 and 3.

\section{Conclusion and future scope}

CPAR-GR is used to develop a classifier with eminent rules that are valuable for health care disease diagnosis and prognosis. Use of CPAR-GR encourages accuracy by $3 \%, 2 \%$ and $7 \%$ than CPAR for heart, pima and sick datasets, whereas it discourages accuracy by $1 \%, 3 \%$ and $3 \%$ for breast cancer, cleve and hepatitis datasets. To enhance the accuracy of CPAR-GR, two different dimensionality reduction techniques such as T-test and reducts are applied over six health care datasets to retain significant attributes by eliminating insignificant ones. From the results, it is evident that, the combination of T-test and CPAR-GR achieves better accuracy for disease prediction compared to CPAR for all datasets except breast cancer. It is inferred that the computation of reducts does not support the performance of CPAR as well as CPAR-GR algorithm compared to T-test. Also, it is found that the computation of reducts after T-test quickly generates single reduct set thereby making reducts computation simple. For experimentation, binary class datasets such as breast cancer, cleve, hepatitis, heart, pima and sick are considered in this paper. In future, multi-class datasets can be focused; other optimal dimensionality reduction techniques can be employed to improve the accuracy of the classifier. Suitable attribute selection measures can also be identified to fit into the CPAR algorithm for building more accurate associative classifiers for multi-class datasets. 


\section{References}

Antonie M L, Zaïane O R and Coman A 2003 Associative classifiers for medical images. Mining Multimedia and Complex Data. Lect. Notes. Comput. Sci. 2797: 68-83

Hassanien A E, Abraham A and Peters J F 2009 Schaefer G Rough sets in medical informatics applications. Adv. Soft. Comp. 2009(58): 23-30

Jabbar M A, Deekshatulu B L and Chandra P 2012 Heart disease prediction system using associative classification and genetic algorithm. In: Proceedings of the International Conference on Emerging Trends in Electrical, Electronics and Communication Technologies, Anantapur, India: Elsevier; 183-192

Jiao N and Miao D 2009 An efficient gene selection algorithm based on tolerance rough set theory. In: Proceedings of the $12^{\text {th }}$ International Conference on Rough Sets, Fuzzy Sets, Data Mining and Granular Computing, Delhi, India: Springer, Berlin Heidelberg; 176-183

Johnson D S 1974 Approximation algorithms for combinatorial problems. J. Comput. Syst. Sci. 9(3): 256278

Kohavi R and Frasca B 1994 Useful feature subsets and rough set reducts. In: Proceedings of the International Workshop on Rough Sets and Soft Computing, San Jose, California, USA: RSSC, USA; 310-317

Komorowski J, Skowron A and Øhrn A 2002 The ROSETTA: rough set software system. In: Kløsgen W and Zytkow J (eds), Handbook of Data Mining and Knowledge Discovery, Oxford: University Press; 554-559

Lei Yu and Huan Liu 2003 Feature selection for high-dimensional data: a fast correlation-based filter solution. In: Proceedings of the International Conference on Machine Learning, Washington, USA: AAAI, USA; 856-863

Li Jiye and Nick Cercone 2005 Discovering and ranking important rules. In: Proceedings of the International Conference on Granular Computing, Beijing, China: IEEE, USA; 506-511

Li S, Liao C and Kwok J T 2006 Gene feature extraction using t-test statistics and kernel partial least squares. In: Proceedings of the International Conference on Neural Information Processing, HongKong, China: Springer, Berlin Heidelberg; 11-20

Li W, Han J and Pei J 2001 CMAR: accurate and efficient classification based on multiple class association rules. In: Proceedings of the International Conference on Data Mining, San Jose, USA: IEEE, USA; 369-376

Liu B, Hsu W and Ma Y 1998 Integrating classification and association rule mining. In: Proceedings of the International Conference on Special Interest Group on Discovery and Data Mining, New York, USA: ACM,USA; 80-86

Noh K, Lee H G, Shon H S, Lee B J and Ryu K H 2006 Associative classification approach for diagnosing cardiovascular disease. Lect. Notes Contr. Inf. 345: 721-727

Øhrn A 1999 Discernibility and rough sets in medicine: tools and applications. Ph.D, Norwegian University of Science and Technology (NTNU), Trondheim, Norway

Pawlak Z 1982 Rough sets. Int. J. Comput. Inf. Sci. 11: 341-356

Pawlak Z 1991 Rough sets: theoretical aspects of reasoning about data. Dordrecht, Boston, London: Kluwer Academic Publishers, 33-78

Pila A D and Monard M C 2001 An empirical comparison of rough sets reducts and other filters approaches for feature subset selection. In: Proceedings of the $6^{\text {th }}$ Iberoamerican Symposium on Pattern Recognition, Florianopolis,SC, Brazil: SIARP,Brazil; 41-49

Poolsawad N, Moore L Kambhampati C and Cleland J G F 2012 Handling missing values in data mining A case study of heart failure dataset. In: Proceedings of the International Conference on Fuzzy Systems and Knowledge Discovery, Sichuan,China: IEEE, USA; 2934-2938

Qiang Shen and Alexios Chouchoulas 2001 Rough set based dimensionality reduction for supervised and unsupervised learning. Int. J. Appl. Math. Comp. 11: 583-601

Quinlan J and Cameron Jones 1993 FOIL: a midterm report. In: Proceedings of the European Conference on Machine Learning, Vienna, Austria, Springer, Berlin Heidelberg; 1-20 
Susmaga R 2004 Tree-like parallelization of reduct and construct computation. In: Proceedings of the International Conference on Rough Sets and Current Trends in Computing, Uppsala, Sweden: Springer, Berlin Heidelberg; 455-464

Vinterbo S and Ohno-Machado L 1999 A genetic algorithm to select variables in logistic regression: example in the domain of myocardial infarction. J. Am. Med. Inform. Assn. 6: 984-988

Wang D, Zhang H, Liu R and Lv W 2012 Feature selection based on term frequency and t-test for text categorization. In: Proceedings of the International Conference on Information and Knowledge Management, Maui, Hawaii, USA: ACM,USA; 1482-1486

Wang L, Chu F and Xie W 2007 Accurate cancer classification using expressions of very few genes. IEEE ACM T Comput. Bi 4: 40-53

Yin X and Han J 2003 CPAR: classification based on predictive association rules. In: Proceedings of the International Conference on Data Mining, San Francisco, USA: SIAM, USA; 331-335 